\title{
DIMENSION OF IMAGES OF SUBSPACES UNDER MAPPINGS IN TRIEBEL-LIZORKIN SPACES
}

\author{
STANISLAV HENCL AND PETR HONZÍK
}

\begin{abstract}
Let $m<\alpha<p$ and let $f: \mathbb{R}^{n} \rightarrow \mathbb{R}^{k}$ be a $s, p$-quasicontinuous representative of a mapping in the Triebel-Lizorkin space $F_{p, q}^{s}$. We find an optimal value of $\beta(n, m, p, \alpha, s)$ such that for $\mathcal{H}^{\beta}$ a.e. $y \in(0,1)^{n-m}$ the Hausdorff dimension of $f\left((0,1)^{m} \times\{y\}\right)$ is at most $\alpha$. We construct examples to show that the value of $\beta$ is optimal and we show that it does not increase once $p$ goes below the critical case $p<\alpha$.
\end{abstract}

\section{INTRODUCTION}

It is well-known that each Sobolev function satisfies the ACL condition, i.e., the function is absolutely continuous when restricted to almost all lines parallel to coordinate axes. It follows that images of $\mathcal{H}^{n-1}$ almost all segments are rectifiable curves and thus have Hausdorff dimension at most one. More generally we can ask how often it can happen that the images of $m$-dimensional subspaces have bigger Hausdorff dimension.

These questions were studied for quasiconformal mapping by Gehring and Väisälä [7] and for supercritical Sobolev mappings (i.e. $f \in W^{1, p}, p>n$ ) by Kaufmann [11]. Let $\Omega \subset \mathbb{R}^{n}$ be a domain, $p>n$ and let $f \in W^{1, p}\left(\Omega, \mathbb{R}^{k}\right)$ be continuous. It was shown by Kaufmann that images of $m$-dimensional subspaces have zero $\mathcal{H}^{\frac{p m}{p-n+m}}$ measure. Let us point out that naive application of $\left(1-\frac{n}{p}\right)$ Hölder continuity would give a worse exponent $\frac{p m}{p-n}$. He also gave a probabilistic construction to show that the value $\frac{p m}{p-n+m}$ is optimal. The generalization of this result to metric spaces setting that include for example Heisenberg group is by Balogh, Tyson and Wildrick [2].

Recently it was shown in a nice paper of Balogh, Monti and Tyson [3] that for any $p>n$ and $m<\alpha<\frac{p m}{p-n+m}$ it is true that the image of $\mathcal{H}^{\beta}$ a.e. $m$-dimensional subspace has dimension at most $\alpha$ where $\beta=n-m-\left(1-\frac{m}{\alpha}\right) p$. By a similar construction as Kaufmann they also showed that this value of $\beta$ is optimal for all $p>n$. Moreover, using finer arguments it is possible to show a similar results also for $p<n$ as was done by Hencl and Honzík [10].

In this paper we show that similar result holds also for higher order Sobolev spaces or more generally for Triebel-Lizorkin spaces $F_{p, q}^{s}$ (see Preliminaries for the definition). Let us just recall that for $k \in \mathbb{N}$ and $p \in(1, \infty)$ we have $F_{p, 2}^{k}=W^{k, p}$. It seems possible that similar result holds also for Besov spaces but we have not pursued this direction.

2000 Mathematics Subject Classification. 46E35, 28A78.

Key words and phrases. Sobolev mapping, Hausdorff dimension.

The authors were supported by the ERC CZ grant LL1203 of the Czech Ministry of Education. 
The only result for fractional Sobolev spaces in this direction that we are aware of is the estimate of the dimension of a graph of a function by Carvalho [5] and Carvalho and Caetano [6]. Our main result in the positive direction is the following.

Theorem 1.1. Let $n, k \in \mathbb{N}$ and $m \in\{1, \ldots, n-1\}$. Let $m<\alpha<p$ and for sp $>n-m$ we moreover assume $\alpha<\frac{p m}{s p-n+m}$. Set

$$
\beta=\beta(\alpha, p, s):=n-m-\left(s-\frac{m}{\alpha}\right) p .
$$

Suppose that $f \in F_{p, q}^{s}\left(\mathbb{R}^{n}, \mathbb{R}^{k}\right)$ is a $s, p$-quasicontinuous representative. If we denote

$$
E=\left\{y \in(0,1)^{n-m}: \operatorname{dim}_{\mathcal{H}}\left(f\left((0,1)^{m} \times\{y\}\right)\right) \geq \alpha\right\},
$$

then $\operatorname{dim}_{\mathcal{H}}(E) \leq \beta$.

Moreover, in the case $s p>n-m$ and $\frac{p m}{s p-n+m}<\alpha<p$ (i.e. $\beta<0$ ) there is no $y \in(0,1)^{n-m}$ such that $\operatorname{dim}_{\mathcal{H}}\left(f\left((0,1)^{m} \times\{y\}\right)\right) \geq \alpha$.

Let us note that sharp results for fractional order spaces $F_{p, q}^{s}$ cannot be obtained from the results for $W^{k, p}$ spaces simply by the Sobolev embedding theorem.

Since the important things occur on a set of measure zero we need to have a good representative of our function. In the theorem, we choose the $s, p$-quasicontinuous representative, but in fact the only thing that we will need is that the value of the representative of $f$ is equal to the limit of integral averages whenever such limit exists.

Analogously to the counterexamples in [11] and [3] we can show that the value of $\beta$ from the previous theorem is optimal at least for Minkowski dimension if $\alpha<p$. For simplicity we give the counterexamples only for $q=2$ but in view of imbeddings (2.3) it would be possible to adjust the construction for other $q$ as well.

Theorem 1.2. Let $s>0,1<p<\infty$ and let $\alpha$ satisfy $m<\alpha \leq \frac{p m}{s p-n+m}$ for $s p>n-m$ and $m<\alpha$ for $s p \leq n-m$, and define

$$
\beta=\beta(\alpha, p, s)=(n-m)-\left(s-\frac{m}{\alpha}\right) p .
$$

Let $E \subset(0,1)^{n-m}$ be any Borel set for which

$$
\limsup _{r \rightarrow 0+} r^{\beta} \mathbf{N}(E, r)<\infty .
$$

Then, for any integer $k>\alpha$, there is a continuous map $f \in F_{p, 2}^{s}\left(\mathbb{R}^{n}, \mathbb{R}^{k}\right)$ so that $f\left(\mathbb{R}^{m} \times\{a\}\right)$ has Hausdorff dimension at least $\alpha$, for $\mathcal{H}^{\beta}$-almost every $a \in E$.

It was shown in [10] that the condition $\alpha<p$ is natural and that better counterexample exists for $W^{1, p}$ spaces if $p<\alpha$. We show below the neccessity of the condition $\alpha<p$ also for Triebel-Lizorkin spaces and our counterxample shows that the optimal value of $\beta$ does not improve once we go below $p<\alpha$. This degeneracy seems to be connected with the fact that $s, p$-quasicontinuous representatives of the function $f \in F_{p, q}^{s}$ are well-defined and have Lebesgue points up to a set of dimension $n-s p$ (see Theorem 2.1 below) and for $p<\alpha$ we have $\beta(\alpha, p, s)<n-s p$.

Theorem 1.3. Let $s>0,1<p<\infty, s p<n, m<p<\alpha$ and let

$$
\tilde{\beta}<n-s p=\beta(p, p, s) \text {. }
$$


Let $E \subset(0,1)^{n-m}$ be any Borel set for which

$$
\limsup _{r \rightarrow 0+} r^{\tilde{\beta}} \mathbf{N}(E, r)<\infty .
$$

Then, for any integer $k>\alpha$, there is a continuous map $f \in F_{p, 2}^{s}\left(\mathbb{R}^{n}, \mathbb{R}^{k}\right)$ so that $f\left(\mathbb{R}^{m} \times\{a\}\right)$ has Hausdorff dimension at least $\alpha$, for $\mathcal{H}^{\tilde{\beta}}$-almost every $a \in E$.

The proof of Theorem 1.1 follows the same principle as the proof in [10] but in addition to the estimate of the oscillation on the small ball, which we used previously, we also need to control the oscilation over larger balls. This is essential in the case $s>1$. We provide these estimates in Lemma 3.1. In the construction of the counterexamples we use the approach developed in [11] and [3] together with the fact that $F_{p, 2}^{s}$ spaces can be obtained by interpolation of Sobolev spaces $W^{k, p}$. For the convenience of the reader we include all details.

\section{Preliminaries}

We use the notation $\mathbf{N}(E, r)$ for the smallest number of balls of radius $r>0$ that cover the set $E \subset \mathbb{R}^{d}$. For $t>0$ we denote the integer part of $t$ as $[t]$. By $Q(z, r)$ we denote the cube centered at $z \in \mathbb{R}^{d}$ with radius $r>0$.

We use the usual convention that $C$ denotes a generic positive constant whose value may change from line to line.

By $f * g$ we denote the convolution

$$
f * g(x)=\int_{\mathbb{R}^{n}} f(x-y) g(y) d y .
$$

The Fourier transform of a function $f$ is denoted as $\hat{f}$ and the inverse Fourier transform is denoted as $\mathcal{F}^{-1}(f)$.

2.1. Fractional Sobolev spaces. Let us recall the Fourier analytic definition of function spaces. We fix a smooth function $\phi$ with $\phi(\xi)=1$ for $|\xi| \leq 1$ and $\phi(\xi)=0$ for $\xi \geq 2$. We then find a function $\psi$ such that $\hat{\psi}(\xi)=\phi(\xi)-\phi(2 \xi)$. We define the operator

$$
\Delta_{j} f=\mathcal{F}^{-1}\left(\hat{f}(\xi) \hat{\psi}\left(2^{-j} \xi\right)\right)=f * \psi_{2^{-j}},
$$

where $\psi_{2^{-j}}(x)=2^{j n} \psi\left(2^{j} x\right)$. This scaling is $L^{1}$ homogeneous. Since $\psi$ is a Schwartz function we know that for every $M>0$ there is $C_{m}>0$ such that $|\psi(x)| \leq C_{M}(1+$ $|x|)^{-M}$. Thus for $k>0$

$$
\sup _{x \in B\left(0,2^{-j+k}\right) \backslash B\left(0,2^{-j+k-1}\right)}\left|\psi_{2^{-j}}(x)\right| \leq C_{M} 2^{j n} 2^{-M k} .
$$

Analogous estimate holds for the derivatives and we obtain for example

$$
\sup _{x \in B\left(0,2^{-j+k}\right) \backslash B\left(0,2^{-j+k-1}\right)}\left|\nabla^{2} \psi_{2^{-j}}(x)\right| \leq C_{M} 2^{2 j} 2^{j n} 2^{-M k} .
$$

The function spaces of interest to us are the Triebel-Lizorkin spaces $F_{p, q}^{s}$ where

$$
\|f\|_{F_{p}^{s, q}}=\left\|\left(\sum_{j=-\infty}^{\infty}\left(2^{s j}\left|\Delta_{j} f\right|\right)^{q}\right)^{\frac{1}{q}}\right\|_{p} .
$$


The norm on domains is defined via restriction. For an introduction to fractional Sobolev spaces, see e.g. [13] and [8, Chapter 6.5.].

The results we prove are independent on the index $q$. This is due to the fact that the following imbeddings are valid:

$$
F_{p, q}^{s} \hookrightarrow F_{p, q+r}^{s} \quad \text { and } \quad F_{p, q+r}^{s+\varepsilon} \hookrightarrow F_{p, q}^{s}
$$

for $r, \varepsilon>0$, see e.g. [9, Proposition 7.2], and we do not pursue the endpoint estimates. We still give the full proofof positive results, because replacing the index $q$ by any single value gives no simplification. We will need that $F_{p, q}^{s}$ functions have Lebesgue points up to a small set. This result follows from [1, Theorem 6.2.1 and Theorem 5.1.13] for the case $q=2$ and extends for other values of $q$ by (2.3).

Theorem 2.1. Let $1<p, q<\infty$ and $0<s p<n$. Let $f \in F_{p, q}^{s}\left(\mathbb{R}^{n}\right)$ be a $s, p$ quasicontinuous representative and set

$$
E_{s, p}=\left\{x \in \mathbb{R}^{n}: x \text { is not a Lebesgue point of } f\right\} .
$$

Then $\operatorname{dim}_{\mathcal{H}}\left(E_{s, p}\right) \leq n-s p$.

2.2. Interpolation of fractional Sobolev spaces. We will need that TriebelLizorkin spaces $F_{p, 2}^{s}$ can be obtained by interpolation of the usual Sobolev spaces (see $\left[4\right.$, Theorem 6.4.5, (7)]). Recall that $F_{p, 2}^{k}=W^{k, p}$ for $k \in \mathbb{N}_{0}$. We use the notation $W^{0, p}$ for the usual $L^{p}$ spaces.

Theorem 2.2. Let $1<p<\infty, k \in \mathbb{N}$, and $k-1 \leq s \leq k$. Let $\theta \in[0,1]$ satisfy $s=\theta(k-1)+(1-\theta) k$. Then for every $f \in C_{c}^{\infty}\left((0,1)^{n}\right)$ we have

$$
\|f\|_{F_{p, 2}^{s}} \leq C\|f\|_{W^{k-1, p}}^{\theta}\|f\|_{W^{k, p}}^{1-\theta} .
$$

In the construction of the counterexamples we will need the estimate of the norms of some simple functions.

Lemma 2.3. Let $p \in(1, \infty)$ and $s \geq 0$. Let us fix $f_{0} \in C_{c}^{\infty}\left(\mathbb{R}^{n}\right)$ such that

$$
f_{0}=1 \text { on } Q_{0}=(0,1)^{n} \text { and } \operatorname{supp} f_{0} \subset 2 Q_{0} \text {. }
$$

A) Let $r \geq 1$. The norm of the function $f_{r}(x)=f_{0}(r x)$ can be estimated by

$$
\left\|f_{r}\right\|_{F_{p, 2}^{s}} \leq C r^{s-\frac{n}{p}}
$$

$B)$ Let us fix $N$ disjoint cubes $Q_{i} \subset(0,1)^{n}$ of side length $r$. Let $\tilde{f}_{i}$ denote the translates of $f_{r}$ such that $\tilde{f}_{i} \equiv 1$ on $Q_{i}$ and $\operatorname{supp} \tilde{f}_{i} \subset 2 Q_{i}$ and choose vectors $\xi_{i}$ in the unit ball of $\mathbb{R}^{k}, k \in \mathbb{N}$. Then for the function $f:(0,1)^{n} \rightarrow \mathbb{R}^{k}$ defined as

$$
f(x)=\sum_{i=1}^{N} \tilde{f}_{i}(x) \xi_{i} \text { we have }\|f\|_{F_{p, 2}^{s}} \leq C N^{\frac{1}{p}}\left\|f_{r}\right\|_{F_{p, 2}^{s}} \leq C N^{\frac{1}{p}} r^{s-\frac{n}{p}}
$$

Proof. As $f_{0}$ is smooth it is easy to see that $f_{r} \in F_{p, 2}^{s}$ and $f \in F_{p, 2}^{s}$. Let us first prove the estimate A) in the case $s \in \mathbb{N} \cup\{0\}$. By the substitution $y=r x$ and

$$
\left\|\nabla^{s} f_{0}\right\|_{L^{p}}=C_{0} \text { we obtain }\left\|\nabla^{s} f_{r}\right\|_{L^{p}}=C_{0} r^{s-\frac{n}{p}},
$$


where $r^{-\frac{n}{p}}$ comes from the Jacobian and $r^{s}$ comes from the derivative of the composition applied $s$-times. As $r \geq 1$ the estimate (2.4) follows for $s \in \mathbb{N} \cup\{0\}$. For general $s$ we can apply Theorem 2.2 and previous estimate to obtain

$$
\left\|f_{r}\right\|_{F_{p, 2}^{s}} \leq C\left(C_{0} r^{k-1-\frac{n}{p}}\right)^{\theta}\left(C_{0} r^{k-\frac{n}{p}}\right)^{(1-\theta)} \leq C r^{s-\frac{n}{p}} .
$$

Now let us prove B) for $s \in \mathbb{N} \cup\{0\}$. The cubes $Q_{i}$ are pairwise disjoint and hence $2 Q_{i}$ have bounded overlap by $2^{n}$. Thus

$$
\left|\sum_{i=1}^{N} f_{i}(x) \xi_{i}\right|^{p} \leq C \sum_{i=1}^{N}\left|f_{i}(x) \xi_{i}\right|^{p} \leq C \sum_{i=1}^{N}\left|f_{i}(x)\right|^{p}
$$

and the similar estimate holds for the derivatives. It follows that

$$
\|f\|_{F_{p, 2}^{s}}^{p} \leq C \sum_{i=1}^{N}\left\|f_{i}\right\|_{F_{p, 2}^{s}}^{p} \leq C N r^{s p-n} .
$$

For general $s$ we can apply Theorem 2.2 and previous estimate to obtain

$$
\|f\|_{F_{p, 2}^{s}} \leq C\left(C N^{\frac{1}{p}} r^{k-1-\frac{n}{p}}\right)^{\theta}\left(C N^{\frac{1}{p}} r^{k-\frac{n}{p}}\right)^{(1-\theta)} \leq C N^{\frac{1}{p}} r^{s-\frac{n}{p}} .
$$

2.3. Probabilistic lemma. In order to prove Theorem 1.2 and Theorem 1.3 we will use a probabilistic approach and we will need the following lemma (see [3, Lemma 4.3] for the proof).

Lemma 2.4. Let $\left\{X_{i}\right\}_{i=1}^{\infty}$ be a countable sequence of independent random variables, identically distributed according to the uniform distribution on the unit ball $B$ in $\mathbb{R}^{k}$. Let $c=\left\{c_{i}\right\} \in \ell_{\infty}$ and finally let $0<\alpha^{\prime}<k+1$. Then there is a constant $C$ which depends only on $k$ and $\alpha^{\prime}$ so that

$$
\mathbb{E}_{\xi}\left(\left|\sum_{i=1}^{\infty} c_{i} X_{i}\right|^{-\alpha^{\prime}}\right) \leq C \rho(c)^{-\alpha^{\prime}}
$$

where $\rho(c)$ denotes the second largest value, i.e.

$$
\rho(c)= \begin{cases}\|c\|_{\infty} & \text { if }\|c\|_{\infty}=\sup _{i \in \mathbb{N}}\left|c_{i}\right| \text { is not attained, } \\ \sup _{i \neq i_{0}}\left|c_{i}\right| & \text { if the supremum is attained at } i_{0} .\end{cases}
$$

2.4. Hausdorff and capacitary dimension. Let $\alpha>0$ and $\varepsilon>0$. We use the usual Hausdorff measure of a set $E \subset \mathbb{R}^{d}$, i.e.

$$
\mathcal{H}_{\varepsilon}^{\alpha}(E)=\inf \left\{\sum_{i=1}^{\infty} \operatorname{diam}^{\alpha} A_{i}: E \subset \bigcup_{i=1}^{\infty} A_{i}, \operatorname{diam} A_{i}<\varepsilon\right\} \text { and } \mathcal{H}^{\alpha}(E)=\lim _{\varepsilon \rightarrow 0+} \mathcal{H}_{\varepsilon}^{\alpha}(E) .
$$

The Hausdorff dimension of a set $E$ is

$$
\operatorname{dim}_{\mathcal{H}}(E)=\sup \left\{\alpha>0: \mathcal{H}^{\alpha}(E)=\infty\right\}=\inf \left\{\alpha>0: \mathcal{H}^{\alpha}(E)=0\right\} .
$$

For $\alpha>0$ and $A \subset \mathbb{R}^{k}$, denote by

$$
I_{\alpha}(\mu):=\int_{A} \int_{A}|x-y|^{-\alpha} d \mu(x) d \mu(y)
$$


the $\alpha$-energy of a nonzero finite Radon measure $\mu$ with compact support in $A$. The capacitary dimension of a set $A$ is defined as

$$
\operatorname{dim}_{c}(A)=\sup \left\{\alpha>0: \exists \mu \text { with } I_{\alpha}(\mu)<\infty\right\} .
$$

We will use the well-known fact (see [12, Theorem 8.9]) that the Hausdorff dimension is equal to the capacitary dimension.

\section{Positive Result}

It is well-known that for $s p>n$ the mappings in $F_{p, q}^{s}$ are Hölder continuous, i.e. for every $a, b \in B\left(0, R_{0}\right)$ we have

$$
|f(a)-f(b)| \leq C R_{0}^{s-\frac{n}{p}}\|f\|_{F_{p, q}^{s}\left(\mathbb{R}^{n}\right)} .
$$

However the similar inequality holds also in the case $s p<n$ for some different ball of radius $R$ if we add the correction term $R / R_{0}$ to some power (see [10, Lemma 3.1] for similar result for $\left.W^{1, p}, p<n\right)$.

Lemma 3.1. Suppose that $a$ and $b$ are Lebesgue points of $f$ in $F_{p}^{s, q}\left(\mathbb{R}^{n}\right), 1 \leq p, q<\infty$ and $s>0$. Let $0<\gamma<p / 2$ and denote $R_{0}=|a-b|$. Then there are $z \in\{a, b\}$ and either $0<R \leq R_{0}$ such that

$$
\int_{B(z, R)}\left(\sum_{j=-\infty}^{\infty}\left(2^{s j}\left|\Delta_{j} f(x)\right|\right)^{q}\right)^{p / q} d x \geq C_{\gamma}|f(a)-f(b)|^{p} R^{n-s p}\left(R / R_{0}\right)^{\gamma}
$$

or $R_{0} \leq R$ such that

$$
\int_{B(z, R)}\left(\sum_{j=-\infty}^{\infty}\left(2^{s j}\left|\Delta_{j} f(x)\right|\right)^{q}\right)^{p / q} d x \geq C_{\gamma}|f(a)-f(b)|^{p} R^{n-s p}\left(R / R_{0}\right)^{p-\gamma} .
$$

where the positive constant $C_{\gamma}$ depends only on $\gamma, p, s$ and dimension $n$.

Proof. Suppose $2^{-k} \leq|a-b|<2^{-k+1}$ for some $k \in \mathbb{Z}$. Since the points $a$ and $b$ are Lebesgue points, we get

$$
\sum_{j \in \mathbb{Z}} \Delta_{j} f(a)=f(a) \text { and } \sum_{j \in \mathbb{Z}} \Delta_{j} f(b)=f(b) .
$$

It follows that by choosing sufficiently small constant $C \approx\left(\sum_{i=1}^{\infty} 2^{-i \gamma}\right)^{-1}$, we get

$$
\left|\Delta_{j} f(a)-\Delta_{j} f(b)\right| \geq C 2^{-|k-j| \gamma / p}|f(a)-f(b)| \text { for some } j \in \mathbb{Z} .
$$

Let us first suppose that $j \geq k$. Without loss of generality we may assume that

$$
\left|\Delta_{j} f(a)\right| \geq C 2^{(k-j) \gamma / p}|f(a)-f(b)| .
$$

Now, let us use the formula

$$
\Delta_{j} f=\left(\Delta_{j-1}+\Delta_{j}+\Delta_{j+1}\right) \Delta_{j} f=\Delta_{j} f * \sum_{i=-1}^{1} \psi_{2^{-j+i}}
$$


This standard identity holds because $\sum_{i=-1}^{1} \hat{\psi}_{2^{-j+i}}(\xi)=1$ for all $\xi \in \operatorname{supp} \hat{\psi}_{2^{-j}}$. We have

$$
\begin{aligned}
\left|\Delta_{j} f(a)\right| \leq C & \int_{B\left(a, 2^{-j}\right)} \sum_{i=-1}^{1}\left|\psi_{2^{-j+i}}(y)\right|\left|\Delta_{j} f(a-y)\right| d y \\
& +\sum_{l=1}^{\infty} \int_{B\left(a, 2^{-j+l}\right) \backslash B\left(a, 2^{-j+l-1}\right)} \sum_{i=-1}^{1}\left|\psi_{2^{-j+i}}(y)\right|\left|\Delta_{j} f(a-y)\right| d y .
\end{aligned}
$$

Now, to handle the first term of the sum we apply the Hölder inequality and (2.1) and we obtain

$\int_{B\left(a, 2^{-j}\right)} \sum_{i=-1}^{1}\left|\psi_{2^{-j+i}}(y)\right|\left|\Delta_{j} f(a-y)\right| d y \leq\left(\int_{B\left(a, 2^{-j}\right)}\left|\Delta_{j} f(a-y)\right|^{p} d y\right)^{\frac{1}{p}} 2^{n j}\left|B\left(a, 2^{-j}\right)\right|^{\frac{1}{p^{\prime}}}$,

while the terms inside the sum are estimated as

$$
\begin{aligned}
& \int_{B\left(a, 2^{-j+l}\right) \backslash B\left(a, 2^{-j+l-1}\right)} \sum_{i=-1}^{1}\left|\psi_{2^{-j+i}}(y)\right|\left|\Delta_{j} f(a-y)\right| d y \\
& \quad \leq C_{M}\left(\int_{B\left(a, 2^{-j+l}\right) \backslash B\left(a, 2^{-j+l-1}\right)}\left|\Delta_{j} f(a-y)\right|^{p} d y\right)^{\frac{1}{p}} 2^{n j-M l}\left|B\left(a, 2^{-j+l}\right)\right|^{\frac{1}{p^{\prime}}} .
\end{aligned}
$$

By the combination of (3.3), (3.5), (3.6) and (3.7) we obtain

$$
\begin{aligned}
2^{(k-j) \gamma / p} \mid & f(a)-f(b) \mid \leq \\
& \leq C_{M} \sum_{l=0}^{\infty}\left(\int_{B\left(a, 2^{-j+l}\right)}\left|\Delta_{j} f(a-y)\right|^{p} d y\right)^{\frac{1}{p}} 2^{n j-M l}\left|B\left(a, 2^{-j+l}\right)\right|^{\frac{1}{p^{\prime}}} \\
& \leq C_{M} \sum_{l=0}^{\infty} 2^{n j-M l} 2^{(-j+l) \frac{n}{p^{\prime}}}\left(\int_{B\left(a, 2^{-j+l}\right)}\left|\Delta_{j} f(a-y)\right|^{p} d y\right)^{\frac{1}{p}} \\
& \leq 4 C_{M} \sup _{l_{0} \in \mathbb{N}_{0}} 2^{n j-(M-1) l_{0}} 2^{\left(-j+l_{0}\right) \frac{n}{p^{\prime}}}\left(\int_{B\left(a, 2^{\left.-j+l_{0}\right)}\right.}\left|\Delta_{j} f(a-y)\right|^{p} d y\right)^{\frac{1}{p}},
\end{aligned}
$$

where in the last step we used the observation that for any sequence $\left\{u_{l}\right\}_{l=0}^{\infty}$ we have

$$
\sum_{l=0}^{\infty} 2^{-l}\left|u_{l}\right| \leq 2 \sup _{l_{0}}\left|u_{l_{0}}\right|
$$

to reduce the sum to one element. The constant $M$ comes from the Schwartz decay formula (2.1) and we can choose $M=3+n-s-\gamma$. Therefore for some $l_{0}$ we have

$$
\begin{aligned}
\int_{B\left(a, 2^{\left.-j+l_{0}\right)}\right.} 2^{s j p}\left|\Delta_{j} f(a-y)\right|^{p} d y & \geq C 2^{s j p} 2^{-n j p+p(n-s+2) l_{0}} 2^{\frac{j n p-l_{0} n p}{p^{\prime}}} 2^{\left(k-j+l_{0}\right) \gamma}|f(a)-f(b)|^{p}, \\
& \geq C R^{n-s p}\left(\frac{R}{R_{0}}\right)^{\gamma} 2^{2 l_{0} p}|f(a)-f(b)|^{p}
\end{aligned}
$$

because we have $2^{k-j+l_{0}} \approx R / R_{0}$ and $R \approx 2^{-j+l_{0}}$. The conclusion (3.1) follows easily and (3.2) follows by $k \leq j$ and $0<\gamma<p / 2$. We note that the result from this part 
would give a stronger estimate than (3.2) with a different choice of $M$, in particular we may get a better power of $R / R_{0}$, but we wish to keep the estimates unified.

In the case $j<k$, we estimate the derivative in order to gain some size over the larger ball. For sufficiently small constant $C \approx\left(\sum_{i=1}^{\infty} 2^{-i \gamma / p}\right)^{-1}$ we get that for some $j \in \mathbb{Z}$ we have

$$
\left|\Delta_{j} f(a)-\Delta_{j} f(b)\right| \geq C 2^{-|k-j| \gamma / p}|f(a)-f(b)| .
$$

By the mean value theorem there is a point $y$ on the line segment $(a, b)$ such that

$$
\left|\nabla \Delta_{j} f(y)\right| \geq C 2^{-|k-j| \gamma / p} 2^{k}|f(a)-f(b)| .
$$

We differentiate the formula (3.4) and we obtain the estimate

$$
\left|\nabla^{2} \Delta_{j} f\right| \leq\left|\Delta_{j} f\right| *\left|\nabla^{2} \sum_{i=-1}^{1} \psi_{2^{-j+i}}\right| .
$$

We assume that the estimate (3.2) fails for some choice of the constant $C_{\gamma}$ and use this assumption to prove it for some other value of $C_{\gamma}$. First we repeat the calculation from (3.5), (3.6) and (3.7) with $\left|\nabla^{2} \sum_{i=-1}^{1} \psi_{2^{-j+i}}\right|$ in place of $\left|\sum_{i=-1}^{1} \psi_{2^{-j+i}}\right|$. This leads to the same result with additional factor $2^{2 j}$ which comes from (2.2). We have for any $z \in B\left(y, 2^{-j}\right)$

$$
\left|\nabla^{2} \Delta_{j} f(z)\right| \leq C_{M} 2^{2 j} \sum_{l=0}^{\infty}\left(\int_{B\left(z, 2^{-j+l}\right)}\left|\Delta_{j} f(z-x)\right|^{p} d x\right)^{\frac{1}{p}} 2^{n j-M l}\left|B\left(z, 2^{-j+l}\right)\right|^{\frac{1}{p^{\prime}}} .
$$

We assumed that the opposite of (3.2) holds for any choice or $R \geq R_{0}$. We use this opposite inequality for $R=2^{-j+l_{0}}$ to estimate the $j$-th term of the sum and we get

$$
\begin{aligned}
\left|\nabla^{2} \Delta_{j} f(z)\right| & \leq C_{M} 2^{2 j} \sup _{l_{0}}\left(\int_{B\left(z, 2^{\left.-j+l_{0}\right)}\right.}\left|\Delta_{j} f(z-y)\right|^{p} d y\right)^{\frac{1}{p}} 2^{n j-(M-1) l_{0}}\left|B\left(z, 2^{-j+l_{0}}\right)\right|^{\frac{1}{p^{\prime}}} \\
& \leq C 2^{2 j}|f(a)-f(b)| 2^{-s j} \sup _{l_{0}} 2^{\left(-j+l_{0}\right) \frac{n-s p}{p}} 2^{\left(-j+l_{0}+k\right) \frac{p-\gamma}{p}} 2^{n j-(M-1) l_{0}} 2^{\left(l_{0}-j\right) \frac{n}{p^{\prime}}} .
\end{aligned}
$$

and so for large value of $M$ the we get

$$
\sup _{x \in B\left(a, 2^{-j}\right)}\left|\nabla^{2} \Delta_{j} f(x)\right| \leq C 2^{j+k} 2^{-|k-j| \frac{\gamma}{p}}|f(a)-f(b)| .
$$

For every $\tilde{x} \in B\left(y, c 2^{-j}\right)$ we use mean value theorem together with (3.9) and (3.8) to obtain

$$
\begin{aligned}
\left|\nabla \Delta_{j} f(y)-\nabla \Delta_{j} f(\tilde{x})\right| & \leq c 2^{-j} \sup _{x \in B\left(a, 2^{-j}\right)}\left|\nabla^{2} \Delta_{j} f(x)\right| \\
& \leq c C 2^{k} 2^{-|k-j| \frac{\gamma}{p}}|f(a)-f(b)| \leq c C\left|\nabla \Delta_{j} f(y)\right| .
\end{aligned}
$$

We see that there is an independent constant $0<c<1$ such that

$$
\sup _{x \in B\left(y, c 2^{-j}\right)}\left|\nabla \Delta_{j} f(y)-\nabla \Delta_{j} f(x)\right| \geq\left|\nabla \Delta_{j} f(y)\right| / 10 .
$$


This means that there exists a direction such that whenever we choose a line parallel to that direction of length of at least $c 2^{-j-10}$ which lies inside of $B\left(y, c 2^{-j}\right)$ on at least one quarter of this line we have with the help of (3.8) and (3.10)

$$
\left|\Delta_{j} f(x)\right| \geq C 2^{k-j} 2^{-|k-j| \frac{\gamma}{p}}|f(a)-f(b)| .
$$

Therefore there is a significant part $P$ of $B\left(a, c 2^{-j}\right)$ such that if $x \in P$, then we get

$$
\left|\Delta_{j} f(x)\right| \geq C 2^{k-j} 2^{-|k-j| \frac{\gamma}{p}}|f(a)-f(b)|
$$

and the claim (3.2) follows from

$$
\int_{B\left(a, c 2^{-j}\right)}\left(2^{s j}\left|\Delta_{j} f(x)\right|\right)^{p} d x \geq C 2^{-j n} 2^{s j p} 2^{(k-j) p} 2^{-(k-j) \gamma}|f(a)-f(b)|^{p} .
$$

Proof of Theorem 1.1. The proof of the Theorem follows the same principle as the proof in [10]. The only difference is additional level of complexity caused by the fact that the estimate given by the Lemma 3.1 is no longer local. There are two points where the proof needs to be altered, first, the balls generated during the proof may overlap significantly and second, it is not possible to limit the diameter of the balls initially. Both of these problems are handled using the additional factor $\left(R / R_{0}\right)^{p}$ in the estimate (3.2).

It is enough to show that $\operatorname{dim}_{\mathcal{H}}(E)<\tilde{\beta}$ for each $\tilde{\beta}>\beta$. Let us fix $\tilde{\beta}>\beta(\alpha, p, s)$ and assume for contradiction that $\operatorname{dim}_{\mathcal{H}}(E) \geq \tilde{\beta}$. By Theorem 2.1 we know that the set

$$
F=\left\{x \in[0,1]^{n}: x \text { is not Lebesgue point of } f\right\}
$$

has Hausdorff dimension at most $n-s p$ and the same holds for its projections. From $p>\alpha$ we know that $\tilde{\beta}>\beta>n-p s$ and hence this set is negligible and

$$
\operatorname{dim}_{\mathcal{H}}(E \backslash P(F)) \geq \tilde{\beta},
$$

where $P$ is the projection on the last $n-m$ variables. By [12, Lemma 3.1 and Theorem 8.13] there is a compact set $E_{0} \subset E \backslash P(F)$ so that $0<\mathcal{H}^{\tilde{\beta}}\left(E_{0}\right)<\infty$. By Frostman's lemma ([12, Theorem 8.9]) we can fix a measure $\mu$ supported in $E_{0}$ with total variation $\|\mu\|=M$, and such that

$$
\mu(B(a, r)) \leq r^{\tilde{\beta}} \text { for any } a \in \mathbb{R}^{n-m} \text { and } r>0 .
$$

We can fix $\tilde{\alpha}<\alpha$ such that $\tilde{\beta}>\beta(\tilde{\alpha}, p, s)$ and $\tilde{\alpha}>m$. It follows that

$$
\mathcal{H}^{\tilde{\alpha}}\left(f\left((0,1)^{m} \times\{y\}\right)\right)=\infty \text { for every } y \in E_{0} .
$$

Now let us fix a huge constant $c_{0}>0$ and let us select $\varepsilon$ such that

$$
\mu\left(E_{1}\right)>\frac{M}{2} \quad \text { for } \quad E_{1}:=\left\{y \in E_{0}: \mathcal{H}_{\varepsilon}^{\tilde{\alpha}}\left(f\left((0,1)^{m} \times\{y\}\right)\right)>c_{0}\right\} .
$$

Fix a point $y \in E_{1}$. Let us introduce dyadic cubes on $[0,1]^{m}$. We denote $\mathcal{D}_{0}=$ $\left\{[0,1]^{m}\right\}$ the mother cube, and $\mathcal{D}_{k}=\left\{Q_{i}\right\}$ where $Q_{i}$ are closed cubes with vertices in the points $2^{-k} \mathbb{Z}^{n} \cap[0,1]^{m}$ and with volume $2^{-k m}$. Simple selection process, described 
in detail in [10] gives us a natural number $K_{y}$ and a sequence of disjoint dyadic cubes $\left\{Q_{y, i}\right\}_{i=1}^{K_{y}}$ such that if we denote $\varepsilon_{i, y}=\operatorname{diam} f\left(Q_{i, y} \times\{y\}\right)$ we have

$$
\sum_{i=1}^{K_{y}} \varepsilon_{i, y}^{\tilde{\alpha}} \geq \frac{c_{0}}{4}
$$

We now fix a constant $\gamma>0$, precise value to be specified later. We fix $C_{\gamma} \approx$ $\sum_{i=0}^{\infty} 2^{-\gamma i}$ and select $k$ such that

$$
\sum_{\left\{i=1, \ldots, K_{y}: Q_{i, y} \in D_{k}\right\}} \varepsilon_{i, y}^{\tilde{\alpha}} \geq C_{\gamma}^{-1} 2^{-\gamma k} \frac{c_{0}}{4} .
$$

For each $Q_{y, i} \in D_{k}$ we may find $a_{y, i}, b_{y, i} \in Q_{y, i}$, where the diam in $\varepsilon_{y, i}$ is almost attained. Now we apply Lemma 3.1 for each $Q_{y, i} \in D_{k}$ and denote the sequence of resulting $n$-dimensional balls $B_{j}$. We categorize the balls according to their diameters and denote

$$
\mathcal{B}_{l}=\left\{B_{j}: 2^{-l} \leq \operatorname{diam}\left(B_{j}\right) \leq 2^{-l+1}\right\} .
$$

For each of these balls we denote the generating cube $Q_{j, y}$ and the appropriate $\varepsilon_{j, y}$. We select $l$ such that

$$
\sum_{\left\{j: B_{j} \in \mathcal{B}_{l}\right\}} \varepsilon_{j, y}^{\tilde{\alpha}} \geq C_{\gamma}^{-2} 2^{-\gamma(k+|k-l|)} \frac{c_{0}}{8} .
$$

Now, we either have $l \geq k$, in which case the balls are essentially disjoint and we may use (3.1) to get

$$
\int_{[0,1]^{m} \times B\left(y, 2^{-l}\right)}\left(\sum_{\kappa=-\infty}^{\infty}\left(2^{s \kappa}\left|\Delta_{\kappa} f(x)\right|\right)^{q}\right)^{\frac{p}{q}} d x \geq C_{\gamma} \sum_{\left\{j: B_{j} \in \mathcal{B}_{l}\right\}} \varepsilon_{j, y}^{p} 2^{-l(n-s p)} 2^{-|k-l| \gamma} .
$$

If $l<k$, the balls have overlap at most of the order $2^{m(k-l)}$, so we use (3.2)

$\int_{[0,1]^{m} \times B\left(y, 2^{-l}\right)}\left(\sum_{\kappa=-\infty}^{\infty}\left(2^{s \kappa}\left|\Delta_{\kappa} f(x)\right|\right)^{q}\right)^{\frac{p}{q}} d x \geq C_{\gamma} \sum_{\left\{j: B_{j} \in \mathcal{B}_{l}\right\}} \varepsilon_{j, y}^{p} 2^{-l(n-s p)} 2^{-m(k-l)} 2^{p(k-l)} 2^{-|k-l| \gamma}$.

Since $p>\alpha$, we use the Hölder inequality

$$
\sum_{\left\{j: B_{j} \in \mathcal{B}_{l}\right\}} \varepsilon_{j, y}^{\tilde{\alpha}} \leq\left(\sum_{\left\{j: B_{j} \in \mathcal{B}_{l}\right\}} \varepsilon_{j, y}^{p}\right)^{\frac{\tilde{\alpha}}{p}}\left(C 2^{k m}\right)^{1-\frac{\tilde{\alpha}}{p}} .
$$

For $l \geq k$ we combine this with (3.13) and with the help of $p>\alpha>\tilde{\alpha}$ we get

$$
\begin{aligned}
\int_{[0,1]^{m} \times B\left(y, 2^{-l}\right)}\left(\sum_{\kappa=-\infty}^{\infty}\left(2^{s \kappa}\left|\Delta_{\kappa} f(x)\right|\right)^{q}\right)^{\frac{p}{q}} d x & \geq C_{\gamma}\left(\sum_{\left\{j: B_{j} \in \mathcal{B}_{l}\right\}} \varepsilon_{j, y}^{\tilde{\alpha}}\right)^{\frac{p}{\tilde{\alpha}}} 2^{-l(n-s p)} 2^{m k\left(1-\frac{p}{\tilde{\alpha}}\right)} 2^{-3 l \gamma} \\
& \geq C_{\gamma}\left(\sum_{\left\{j: B_{j} \in \mathcal{B}_{l}\right\}} \varepsilon_{j, y}^{\tilde{\alpha}}\right)^{\frac{p}{\tilde{\alpha}}} 2^{-l(n-s p)} 2^{m l\left(1-\frac{p}{\tilde{\alpha}}\right)} 2^{-3 l \gamma} .
\end{aligned}
$$


The same inequality in fact holds also for $l<k$. In this case we use (3.14) to estimate the left hand side by

$$
\begin{gathered}
C_{\gamma}\left(\sum_{\left\{j: B_{j} \in \mathcal{B}_{l}\right\}} \varepsilon_{j, y}^{\tilde{\alpha}}\right)^{\frac{p}{\tilde{\alpha}}} 2^{-l(n-s p)} 2^{-m(k-l)} 2^{p(k-l)} 2^{m k\left(1-\frac{p}{\tilde{\alpha}}\right)} 2^{-|k-l| \gamma} \geq \\
\geq C_{\gamma}\left(\sum_{\left\{j: B_{j} \in \mathcal{B}_{l}\right\}} \varepsilon_{j, y}^{\tilde{\alpha}}\right)^{\frac{p}{\tilde{\alpha}}} 2^{-l(n-s p)} 2^{m l\left(1-\frac{p}{\tilde{\alpha}}\right)} 2^{-3 l \gamma}
\end{gathered}
$$

where in the last step we use $l<k$ and $p-m+m\left(1-\frac{p}{\tilde{\alpha}}\right)>0$ (by $\tilde{\alpha}>m$ ) and this neglected factor also allows to pass from $2^{-|k-l| \gamma}$ to $2^{-3 l \gamma}$ for small enough $\gamma$.

For each $y$, we have a centered ball in the space $[0,1]^{n-m}$ of diameter $2^{-l_{y}}$. We use Besicovitch theorem to select a disjoint collections $B\left(y_{\nu}, 2^{-l_{\nu}}\right)$ from these. Using (3.12) we obtain that for one of these subcollections we have $\mu\left(\cup B\left(y_{\nu}, 2^{-l_{\nu}}\right)\right) \geq$ $C(n) M$ where the constant involves the constant from the Besicovitch theorem. Finally, we pass to a subcollection $B\left(y_{\nu}, 2^{-l}\right)$, where $l$ is fixed and for some $C \approx$ $C(n)\left(\sum_{l=0}^{\infty} 2^{-\gamma l}\right)^{-1}$ we have

$$
\mu\left(\bigcup B\left(y_{\nu}, 2^{-l}\right)\right) \geq C M 2^{-\gamma l} .
$$

By (3.11) we conclude that the size of the index set $N=\{\nu\}$, which indexes this subcollection is at least $2^{\tilde{\beta} l} C M 2^{-\gamma l}$. Using (3.16) we then write

$$
\begin{aligned}
\|f\|_{F_{p, q}^{s}}^{p} & \geq \sum_{\nu \in N} \int_{[0,1]^{m} \times B\left(y_{\nu}, 2^{-l}\right)}\left(\sum_{\kappa=-\infty}^{\infty}\left(2^{s \kappa}\left|\Delta_{\kappa} f(x)\right|\right)^{q}\right)^{\frac{p}{q}} d x \\
& \geq 2^{\tilde{\beta} l} C M 2^{-\gamma l} 2^{-l(n-s p)} 2^{-3 l \gamma} 2^{l m\left(1-\frac{p}{\tilde{\alpha}}\right)}\left(\sum_{\left\{j: B_{j} \in \mathcal{B}_{l}\right\}} \varepsilon_{j, y}^{\tilde{\alpha}}\right)^{\frac{p}{\tilde{\alpha}}} \\
& \geq C M 2^{\tilde{\beta} l} 2^{-4 \gamma l} 2^{-l(n-s p)} 2^{l m\left(1-\frac{p}{\tilde{\alpha}}\right)} c_{0}^{\frac{p}{\tilde{\alpha}}} .
\end{aligned}
$$

Since $\tilde{\beta}>\beta(\tilde{\alpha}, p, s)=n-m-\left(s-\frac{m}{\tilde{\alpha}}\right) p$, we see that if $\gamma$ is small, the expression above is bounded from below by a multiple of $c_{0}^{\frac{p}{\tilde{\alpha}}}$. Moreover, the constant $c_{0}$ may be chosen arbitrarily large, leading to a contradiction with the finiteness of the norm of $f$.

The case $s p>n-m$ and $\frac{p m}{s p-n+m}<\alpha<p$ can be treated analogously. These inequalities imply $s p>n$ and hence we can choose a continuous representative of $f$. We choose $\alpha>\tilde{\alpha}>\frac{p m}{s p-n+m}$ and for contrary we assume that there is $y$ with

$$
\mathcal{H}^{\tilde{\alpha}}\left(f\left((0,1)^{m} \times\{y\}\right)\right)=\infty .
$$

We again have inequality (3.16) and by $m\left(1-\frac{p}{\tilde{\alpha}}\right)-(n-s p)>0$ the right hand side is bigger than a constant multiple of $c_{0}^{\frac{p}{\tilde{\alpha}}}$ which again leads to a contradiction.

\section{Counterexamples}

First we prove Theorem 1.3. We use the approach that was developed in [3, Theorem 1.4] and [11, Theorem 3]. In the proof we need a measure on the image of $m$-dimensional hyperplanes (pushforward of the $m$-dimensional Hausdorff measure) 
and then we use the definition of capacitary dimension which equals the Hausdorff dimension. For the convenience of the reader we include all details.

Proof of Theorem 1.2. Note that the inequality $\alpha \leq \frac{p m}{s p-n+m}$ for $s p>n-m$ implies that $\beta>0$ while $\beta>0$ always holds for $s p \leq n-m$.

Let us denote the orthogonal splitting of $\mathbb{R}^{n}$ by

$$
V=\mathbb{R}^{m} \times\{0\}^{n-m} \text { and } \quad V^{\perp}=\{0\}^{m} \times \mathbb{R}^{n-m}
$$

and for $a \in \mathbb{R}^{n}$ we denote $V_{a}=V+a$. We assume that our set $E$ satisfies

$$
\mathbf{N}(E, r) \leq C r^{-\beta} \text {. }
$$

We will construct a map $f \in F_{p, 2}^{s}\left(\mathbb{R}^{n}, \mathbb{R}^{k}\right)$ that satisfies

$$
\mathcal{H}^{\alpha^{\prime}}\left(f_{\xi}\left(V_{a} \cap[0,1]^{n}\right)\right)=\infty
$$

for $\mathcal{H}^{\beta}$ almost every $a \in E$ and almost surely in $\xi$, for each $\alpha^{\prime}<\alpha$.

Let us introduce the sets that will serve as a set of indices in our construction. Denote $W=\left\{1, \ldots, 2^{n}\right\}$ and let $W^{j}$ be the set of (ordered) $j$-tuples of elements of $W$ and let

$$
W^{*}=\bigcup_{j \geq 0} W^{j}
$$

We say that $w=\left(w_{1}, \ldots, w_{k}\right)$ is a subword of $v=\left(v_{1}, \ldots, v_{j}\right)$ if $j \geq k$ and $v_{i}=w_{i}$ for $i=1, \ldots, k$. The length of a word $w \in W^{j}$ is equal to $j$ and we denote it as $|w|$. We use the set $W^{*}$ to index the cubes in the standard dyadic decomposition

$$
\mathcal{D}=\left\{Q_{w}\right\}_{w \in W^{*}}
$$

of $Q=[0,1]^{n}$. It follows that the side length of $Q_{w}$ is equal to $2^{-j}$ if $w \in W^{j}$ and that $Q_{v} \subset Q_{w}$ if $w$ is a subword of $v$. We project these cubes into the subspaces $V$ and $V^{\perp}$ and we denote

$$
Q_{w}^{V^{\perp}}=P_{V^{\perp}}\left(Q_{w}\right) \quad \text { and } \quad Q_{w}^{V}=P_{V}\left(Q_{w}\right)
$$

where $P_{V}$ and $P_{V^{\perp}}$ are the corresponding projections. Analogously to the definition of $W^{j}$ we can define a system of $2^{j m}$ dyadic cubes in $[0,1]^{m}$ and we denote this system as $\tilde{W}^{j}$. To simplify the notation we write

$$
W^{j}(E)=\left\{w \in W^{j}: Q_{w}^{V^{\perp}} \cap E \neq \emptyset\right\} .
$$

Let us fix $f_{0} \in C_{c}^{\infty}\left(\mathbb{R}^{n}\right)$ such that $f_{0}=1$ on $Q_{0}=(0,1)^{n}$ and $\operatorname{supp} f_{0} \subset 2 Q_{0}$. For each $w \in W^{*}$, let $\psi_{w} \in C_{0}^{\infty}\left(\mathbb{R}^{n}\right)$ be a function defined as a proper translate of $f_{2^{|w|}}$ from Lemma 2.3. That is $\psi_{w}$ is supported in the cube $2 Q_{w}$ of side length $22^{-w}$ and

(i) $0 \leq \psi_{w} \leq 1$,

(ii) $\psi_{w} \equiv 1$ on $Q_{w}$,

(iii) $\psi_{w} \equiv 0$ on the complement of $2 Q_{w}$,

(iv) $\left\|\psi_{w}\right\|_{F_{p, 2}^{s}} \leq C\left(2^{|w|}\right)^{s-\frac{n}{p}}$. 
Let $\xi=\left\{\xi_{w}\right\}_{w \in W^{*}}$ be a countable sequence of elements from the unit ball in $\mathbb{R}^{k}$. For each $j \geq 1$ we define

$$
f_{\xi, j}=\sum_{w \in W^{j}(E)} \frac{1}{j^{2}} 2^{-\frac{m j}{\alpha}} \psi_{w}(a, x) \xi_{w}, \text { for } x \in V, a \in V^{\perp}
$$

and finally we set

$$
f_{\xi}=\sum_{j=1}^{\infty} f_{\xi, j}
$$

Since

$$
\left\|f_{\xi, j}\right\|_{L^{\infty}} \leq C \frac{1}{j^{2}} 2^{-\frac{m j}{\alpha}}
$$

it is easy to see that $f_{\xi}$ is continuous.

We have $2^{j m} \times 2^{j(n-m)}$ cubes $Q_{w}$ for $w \in W^{j}$ and we have to estimate the number of such a cubes whose projection intersects $E$. By (4.1) we know that we can cover $E$ by $C 2^{j \beta}$ balls of radius $2^{-(j+1)}$ and each of these balls can be covered by at most $2^{n-m}$ dyadic cubes of side length $2^{-j}$. It follows that the number of cubes $Q_{w}$ for $w \in W^{j}(E)$ can be estimated from above by

$$
N=C 2^{j m} \times 2^{j \beta} .
$$

The cubes $Q_{w}, w \in W^{j}(E)$, are disjoint and the norm of the function $j^{2} 2^{\frac{m j}{\alpha}} f_{\xi, j}$ can be estimated by Lemma $2.3 \mathrm{~B}$ ) and hence

$$
\left\|f_{\xi, j}\right\|_{F_{p, 2}^{s}} \leq C \frac{1}{j^{2}} 2^{-\frac{m j}{\alpha}} N^{\frac{1}{p}}\left(2^{j}\right)^{s-\frac{n}{p}} \leq \frac{C}{j^{2}} 2^{-\frac{m j}{\alpha}} 2^{\frac{j m}{p}} 2^{j\left(\frac{\beta}{p}-\frac{n}{p}+s\right)}=\frac{C}{j^{2}} .
$$

where we have used $\beta=(n-m)-\left(s-\frac{m}{\alpha}\right) p$ in the last step. By summing up we obtain that $f_{\xi} \in F_{p, 2}^{s}\left(\mathbb{R}^{n}, \mathbb{R}^{k}\right)$ as

$$
\left\|f_{\xi}\right\|_{F_{p, 2}^{s}} \leq \sum_{j=1}^{\infty}\left\|f_{\xi, j}\right\|_{F_{p, 2}^{s}} \leq C \sum_{j=1}^{\infty} \frac{1}{j^{2}}<\infty .
$$

In the remaining part of the proof we would like to show that for a generic choice of $\xi$ we obtain a map $f_{\xi}$ with the desired property (4.2). Let us view $\xi=\left\{\xi_{w}\right\}_{w \in W^{*}}$ as a sequence of independent random variables, identically distributed according to the uniform probability distribution on the unit ball $B$ in $\mathbb{R}^{k}$. Since Hausdorff and capacitary dimension coincide (see Preliminaries) it is now enough to show that for each $\alpha^{\prime}<\alpha$ we can find a measure $\mu$ on $f_{\xi}\left([0,1]^{m} \times\{a\}\right)$ with finite $\alpha^{\prime}$-energy.

For each $a \in E$ consider the measure $\left(f_{\xi}\right)_{\#}\left(\left.\mathcal{H}^{m}\right|_{[0,1]^{m} \times\{a\}}\right)$, i.e. the pushforward of the $\mathcal{H}^{m}$-measure on $[0,1]^{m} \times\{a\}$ via the map $f_{\xi}$. Let us denote $\mathcal{H}_{a}^{m}=\left.\mathcal{H}^{m}\right|_{[0,1]^{m} \times\{a\}}$. We claim that the expectation

$$
\mathbb{E}_{\xi}\left(\int_{E} I_{\alpha^{\prime}}\left(\left(f_{\xi}\right)_{\#}\left(\mathcal{H}_{a}^{m}\right)\right) d \mathcal{H}^{\beta}(a)\right)
$$

is finite for each $\alpha^{\prime}<\alpha$. It follows that almost surely with respect to $\xi$ we obtain that

$$
I_{\alpha^{\prime}}\left(\left(f_{\xi}\right)_{\#}\left(\mathcal{H}_{a}^{m}\right)\right) \text { is finite for } \mathcal{H}^{\beta} \text { a.e. } a \in E
$$

and our conclusion follows once we prove the claim (4.3). 
Using Fubini theorem we may transform the integral from (4.3) to

$$
\int_{[0,1]^{m}} \int_{[0,1]^{m}} \int_{E} \mathbb{E}_{\xi}\left(\left|f_{\xi}(a, x)-f_{\xi}(a, y)\right|^{-\alpha^{\prime}}\right) d \mathcal{H}^{\beta}(a) d \mathcal{H}_{a}^{m}(x) d \mathcal{H}_{a}^{m}(y) .
$$

We write

$$
f_{\xi}(a, x)-f_{\xi}(a, y)=\sum_{w \in W^{*}(E)} c_{w}(a, x, y) \xi_{w}
$$

where the coefficients are given by

$$
c_{w}(a, x, y)=\frac{1}{|w|^{2}} 2^{-\frac{m|w|}{\alpha}}\left(\psi_{w}(a, x)-\psi_{w}(a, y)\right) .
$$

Let us fix $a \in E$ and $y \in[0,1]^{m} \times\{a\}$. The sequence of the coefficients $c$ clearly belongs to $\ell^{\infty}$ and thus we may use Lemma 2.4 and our task is reduced to the proof of

$$
\int_{[0,1]^{m}} \rho(c(a, x, y))^{-\alpha^{\prime}} d \mathcal{H}_{a}^{m}(x) \leq C<\infty .
$$

where the constant $C$ is independent of $a$ and $y$. For $x \in[0,1]^{m} \times\{a\}$ let us denote by $j(x)$ the largest integer such that both $x$ and $y$ lie in the same $Q_{w} \ni x, y$ for $w \in W^{j(x)}$. It follows that they lie in different $Q_{u_{1}} \ni x$ and $Q_{u_{2}} \ni y$ for $u_{1}, u_{2} \in W^{j(x)+1}$. It follows that most terms in (4.4) and (4.5) cancel and the first nonzero term corresponds to $j(x)+1$. Since $\psi_{w}(a, x)=1$ on $Q_{u_{1}}$ and $\psi_{w}(a, x)=0$ on the complement of $2 Q_{u_{2}}$ it is easy to see that the supremum norm of the difference of these two functions is 1. We can do similar observation for the term $j(x)+2$ which must be again nonzero and hence we obtain

$$
\|c(a, x, y)\|_{\infty}=\frac{1}{(j(x)+1)^{2}} 2^{-\frac{m(j(x)+1)}{\alpha}} \text { and } \rho(c(a, x, y))=\frac{1}{(j(x)+2)^{2}} 2^{-\frac{m(j(x)+2)}{\alpha}} .
$$

From the construction of $W^{j}$ we know that for each $j=j(x)$ we have a fixed cube $Q_{u_{2}} \ni y$ and we can find at most $2^{m}-1$ cubes $Q_{u_{1}}$ such that $x \in Q_{u_{1}}$ and hence

$$
\mathcal{H}_{a}^{m}\left(\left\{x \in[0,1]^{m} \times\{a\}: j(x)=j\right\}\right)=\left(2^{m}-1\right) \mathcal{H}_{a}^{m}\left(Q_{u_{2}}\right)=\left(2^{m}-1\right) 2^{-m(j+1)} .
$$

Now we can estimate

$$
\int_{[0,1]^{m}} \rho(c(a, x, y))^{-\alpha^{\prime}} d \mathcal{H}_{a}^{m}(x) \leq \sum_{j=0}^{\infty}\left(2^{m}-1\right) 2^{-m(j+1)} \frac{1}{(j+2)^{2}} 2^{m(j+2) \frac{\alpha^{\prime}}{\alpha}} .
$$

Since $\alpha^{\prime}<\alpha$ it is easy to see that the series converges which finishes our proof.

Now we proceed to the proof of Theorem 1.3. In contrast with the previous construction (or the construction from [3]) we do not put some basic function into each subcube that intersects our set but only into some of them. Instead of the pushforward of the $m$-dimensional Hausdorff measure we need to use the pushforward of the natural measure on the Cantor type set that is created as the intersection of the subcubes from our construction. 
Proof of Theorem 1.3. We use the notation $V, V^{\perp}, W^{j}, \tilde{W}^{j}$ and so on introduced in the previous proof.

In $W^{j}$ we have $2^{j n}=2^{j m} \times 2^{j(n-m)}$ cubes and we would like to define $W_{G}^{j} \subset W^{j}$ with $2^{[\sqrt{j}] m} \times 2^{j(n-m)}$ cubes $Q_{w}$ for $w \in W_{G}^{j}$. We first choose $2^{[\sqrt{j}] m}$ cubes from $\tilde{W}^{j}$ and then we choose all cubes $Q_{w}, w \in W^{j}$, such that $Q_{w}^{V}$ lies in this system $\tilde{W}^{j}$. Our only requirements for the position of these cubes are that

a) for each $w \in W_{G}^{j}$ there is $v \in W_{G}^{j-1}$ such that $Q_{w} \subset Q_{v}$,

b) for each $w \in W_{G}^{j}$ there are at most $2^{m}$ pairwise essentially disjoint cubes $Q_{u_{i}} \in W_{G}^{j+1}$ such that $Q_{u_{i}} \subset Q_{w}$,

c) number of different cubes in $\left\{Q^{V^{\perp}}, w \in W_{G}^{j}\right\}$ is $2^{j(n-m)}$.

The construction of this system of cubes is given with details in [10].

To simplify the notation we write

$$
W_{G}^{j}(E)=\left\{w \in W_{G}^{j}: Q_{w}^{V^{\perp}} \cap E \neq \emptyset\right\} .
$$

The cubes from $W_{G}^{j}$ naturally form a Cantor type set in $\mathbb{R}^{m}$

$$
G:=\bigcap_{j=1}^{\infty} \bigcup_{w \in W_{G}^{j}} Q_{w}^{V} .
$$

Let us fix $f_{0} \in C_{c}^{\infty}\left(\mathbb{R}^{n}\right)$ such that $f_{0}=1$ on $Q_{0}=(0,1)^{n}$ and $\operatorname{supp} f_{0} \subset 2 Q_{0}$. For each $w \in W^{*}$, let $\psi_{w} \in C_{0}^{\infty}\left(\mathbb{R}^{n}\right)$ be a function defined as in the previous proof. Set $W_{G}^{*}=\bigcup_{j \geq 0} W_{G}^{j}$. Let $\xi=\left\{\xi_{w}\right\}_{w \in W_{G}^{*}}$ be a countable sequence of elements from the unit ball in $\mathbb{R}^{k}$. For each $j \geq 1$ we define

$$
f_{\xi, j}=\sum_{w \in W_{G}^{j}(E)} 2^{-\frac{m[\sqrt{3}]}{\alpha}} \psi_{w}(a, x) \xi_{w}, \text { for } x \in V, a \in V^{\perp}
$$

and again we set

$$
f_{\xi}=\sum_{j=1}^{\infty} f_{\xi, j}
$$

By

$$
\left\|f_{\xi, j}\right\|_{L^{\infty}} \leq C 2^{-\frac{m[\sqrt{j}]}{\alpha}}
$$

it is easy that $f_{\xi}$ is continuous.

We have $2^{[\sqrt{j}] m} \times 2^{j(n-m)}$ cubes $Q_{w}$ for $w \in W_{G}^{j}$ and we have to estimate the number of such a cubes whose projection intersects $E$. From the construction of $W_{G}^{j}$ c) we know that the number of cubes projected to $V^{\perp}$ is $2^{j(n-m)}$, that is all dyadic cubes are available for our covering. By (4.1) we know that we can cover $E$ by $C 2^{j \tilde{\beta}}$ balls of radius $2^{-(j+1)}$ and each of these balls can be covered by at most $2^{n-m}$ dyadic cubes of side length $2^{-j}$. It follows that the number of cubes $Q_{w}$ for $w \in W_{G}^{j}(E)$ can be estimated from above by

$$
N=C 2^{[\sqrt{j}] m} \times 2^{j \tilde{\beta}} .
$$


The cubes $Q_{w}, w \in W_{G}^{j}(E)$, are disjoint and the norm of the function $2^{\frac{m[\sqrt{3}]}{\alpha}} f_{\xi, j}$ can be estimated by Lemma $2.3 \mathrm{~B})$ and hence

$$
\left\|f_{\xi, j}\right\|_{F_{p, 2}^{s}} \leq C 2^{-\frac{m[\sqrt{3}]}{\alpha}} N^{\frac{1}{p}}\left(2^{j}\right)^{s-\frac{n}{p}} \leq C 2^{-\frac{m[\sqrt{3}]}{\alpha}} 2^{\frac{[\sqrt{3}] m}{p}} 2^{j\left(\frac{\tilde{\beta}}{p}-\frac{n}{p}+s\right)} .
$$

Since $\tilde{\beta}<n-s p$ it is easy to see that $f_{\xi} \in F_{p, 2}^{s}\left(\mathbb{R}^{n}, \mathbb{R}^{k}\right)$ by

$$
\left\|f_{\xi}\right\|_{F_{p, 2}^{s}} \leq \sum_{j}\left\|f_{\xi, j}\right\|_{F_{p, 2}^{s}} \leq C \sum_{j} 2^{\frac{(\tilde{\beta}-n+s p)}{p}} 2^{-[\sqrt{j}] m\left(\frac{p}{\alpha}-1\right) \frac{1}{p}}<\infty .
$$

In the remaining part of the proof we would like to show that for a generic choice of $\xi$ we obtain a map $f_{\xi}$ with the desired property (4.2). Let us view $\xi=\left\{\xi_{w}\right\}_{w \in W_{G}^{*}}$ as a sequence of independent random variables, identically distributed according to the uniform probability distribution on the unit ball $B$ in $\mathbb{R}^{k}$. Instead of the conclusion (4.2) we will even show that

$$
\mathcal{H}^{\alpha^{\prime}}\left(f_{\xi}\left(G_{a} \cap[0,1]^{n}\right)\right)=\infty
$$

where $G_{a}$ is a Cantor type set in $V_{a}$ constructed as in (4.6). It is now enough to show that for each $\alpha^{\prime}<\alpha$ we can find a measure $\mu$ on $f_{\xi}\left(G_{a} \cap[0,1]^{n}\right)$ with finite $\alpha^{\prime}$-energy.

On the Cantor type set $G_{a}$ there is a natural measure $\mathcal{H}_{G_{a}}$ such that

$$
\mathcal{H}_{G_{a}}\left(Q_{w}\right)=\frac{1}{\# W_{G}^{j}}=2^{-m[\sqrt{j}]} \quad \text { for each } \quad w \in W_{G}^{j} .
$$

The existence of such a measure was sketched in [10].

For each $a \in E$ consider the measure $\left(f_{\xi}\right)_{\#}\left(\mathcal{H}_{G_{a}}\right)$, i.e. the pushforward of the $\mathcal{H}_{G_{a}}$-measure on $G_{a}$ via the map $f_{\xi}$. This measure is nonzero, because the set $G_{a}$ is nonempty. We claim that the expectation

$$
\mathbb{E}_{\xi}\left(\int_{E} I_{\alpha^{\prime}}\left(\left(f_{\xi}\right)_{\#}\left(\mathcal{H}_{G_{a}}\right)\right) d \mathcal{H}^{\tilde{\beta}}(a)\right)
$$

is finite for each $\alpha^{\prime}<\alpha$. It follows that almost surely with respect to $\xi$ we obtain that

$$
I_{\alpha^{\prime}}\left(\left(f_{\xi}\right)_{\#}\left(\mathcal{H}_{G_{a}}\right)\right) \text { is finite for } \mathcal{H}^{\tilde{\beta}} \text { a.e. } a \in E
$$

and our conclusion follows once we prove the claim (4.9).

Using Fubini theorem we may transform the integral from (4.9) to

$$
\int_{[0,1]^{m}} \int_{[0,1]^{m}} \int_{E} \mathbb{E}_{\xi}\left(\left|f_{\xi}(a, x)-f_{\xi}(a, y)\right|^{-\alpha^{\prime}}\right) d \mathcal{H}^{\tilde{\beta}}(a) d \mathcal{H}_{G_{a}}(x) d \mathcal{H}_{G_{a}}(y)
$$

We write

$$
f_{\xi}(a, x)-f_{\xi}(a, y)=\sum_{w \in W_{G}^{*}(E)} c_{w}(a, x, y) \xi_{w}
$$

where the coefficients are given by

$$
c_{w}(a, x, y)=2^{-\frac{m[\sqrt{|w|}]}{\alpha}}\left(\psi_{w}(a, x)-\psi_{w}(a, y)\right) .
$$


Let us fix $a \in E$ and $y \in G_{a}$. The sequence of the coefficients $c$ clearly belongs to $\ell^{\infty}$ and thus we may use Lemma 2.4 and our task is reduced to the proof of

$$
\int_{[0,1]^{m}} \rho(c(a, x, y))^{-\alpha^{\prime}} d \mathcal{H}_{G_{a}}(x) \leq C<\infty .
$$

where the constant $C$ is independent of $a$ and $y$. For $x \in G_{a}$ let us denote by $j(x)$ the largest integer such that both $x$ and $y$ lie in the same $Q_{w} \ni x, y$ for $w \in W_{G}^{j(x)}$. Similarly to the previous proof we have

$$
\|c(a, x, y)\|_{\infty}=2^{-\frac{m[\sqrt{j(x)+1}]}{\alpha}} \text { and } \rho(c(a, x, y))=2^{-\frac{m[\sqrt{j(x)+2}]}{\alpha}} .
$$

From the construction of $W_{G}^{j}$ part b) we know that for each $j=j(x)$ we have a fixed cube $Q_{u_{2}} \ni y$ and we can find at most $2^{m}-1$ cubes $Q_{u_{1}}$ such that $x \in Q_{u_{1}}$ and hence

$$
\mathcal{H}_{G_{a}}\left(\left\{x \in G_{a}: j(x)=j\right\}\right)=\left(2^{m}-1\right) \mathcal{H}_{G_{a}}\left(Q_{u_{2}}\right)=\left(2^{m}-1\right) 2^{-m[\sqrt{j+1}]} .
$$

Now we can estimate

$$
\int_{[0,1]^{m}} \rho(c(a, x, y))^{-\alpha^{\prime}} d \mathcal{H}_{G_{a}}(x) \leq \sum_{j=0}^{\infty}\left(2^{m}-1\right) 2^{-m[\sqrt{j+1}]} 2^{m[\sqrt{j+2}] \frac{\alpha^{\prime}}{\alpha}} .
$$

Since $\alpha^{\prime}<\alpha$ it is easy to see that the series converges which finishes our proof.

\section{REFERENCES}

[1] D. R. Adams, L. I. Hedberg, Function spaces and potential theory, Springer-Verlag, Berlin.

[2] Z.M. Balogh, J.T. Tyson, K. Wildrick, Dimension distortion by Sobolev mappings in foliated metric spaces, preprint 2012.

[3] Z.M. Balogh, R. Monti, J.T. Tyson, Frequency of Sobolev and quasiconformal dimension distortion, to appear in J. Math. Pures Appl.

[4] J. Bergh, J. Löfström, Interpolation spaces. An introduction, Springer-Verlag, Berlin-New York.

[5] A. Carvalho, Box dimension, oscillation and smoothness in function spaces, J. Funct. Spaces Appl. 3 no. 3 (2005), 287-320.

[6] A. Carvalho, A. Caetano, On the Hausdorff dimension of continuous functions belonging to Hölder and Besov spaces on fractal $d$-sets, J. Fourier Anal. Appl. 18 no. 2 (2012), 386-409.

[7] F. W. Gehring, J. Väisälä, Hausdorff dimension and quasiconformal mappings, J. London Math. Soc. 6 no. 2 (1973), 504-512.

[8] L. Grafakos, Modern Fourier analysis, Second edition. Graduate Texts in Mathematics, 250. Springer, New York.

[9] D. D. Haroske, Envelopes and sharp embeddings of function spaces, Chapman \& Hall/CRC Research Notes in Mathematics, 437.

[10] S. Hencl, P. Honzík, Dimension of images of subspaces under Sobolev mappings, Ann. Inst. H. Poincaré Anal. Non Linéaire 29 no. 3 (2012), 401-411.

[11] R. Kaufman, Sobolev spaces, dimension, and random series, Proc. Amer. Math. Soc. 128 no. 2 (2000), 427-431.

[12] P. Mattila, Geometry of sets and measures in Euclidean spaces, 44, Cambridge Studies in Advanced Mathematics, Cambridge University Press.

[13] H. Triebel, Theory of function spaces, Monographs in Mathematics, 78. Birkhäuser Verlag, Basel.

[14] W. P. Ziemer, Weakly differentiable functions. Graduate texts in Mathematics, 120, SpringerVerlag. 
Department of Mathematical Analysis, Charles University, Sokolovská 83, 18600 Prague 8, Czech Republic

E-mail address: hencl@karlin.mff.cuni.cz

E-mail address: honzik@gmail.com 